\title{
Analysis of FMR1 (CGG) alleles and DXS548-FRAXAC1 haplotypes in three European circumpolar populations: traces of genetic relationship with Asia
}

\author{
Lars Allan Larsen ${ }^{* 1,4}$, Jens Vuust ${ }^{1}$, Mona Nystad ${ }^{2}$, Irina Evseeva ${ }^{3}$, Marijke Van Ghelue ${ }^{2}$ and \\ Lisbeth Tranebjærg*,2,5,6
}

${ }^{1}$ Department of Clinical Biochemistry, Statens Serum Institut, Artillerivej 5, DK-2300 Copenhagen, Denmark;

${ }^{2}$ Department of Medical Genetics, University Hospital, N-9038 Troms $\emptyset$, Norway; ${ }^{3}$ HLA laboratory, Department of Immunology, Arkhangelsk Medical Institute, Arkhangelsk, Russia

Fragile $\mathrm{X}$ syndrome, the most common form of inherited mental retardation, is caused by expansion of a $(C G G)_{n}$ repeat located in the FMR1 gene. The molecular factors involved in the mutation process from stable (CGG) alleles towards unstable alleles are largely unknown, although family transmission studies and population studies have suggested that loss of AGG interruptions in the (CGG) repeat is essential. We have analysed the AGG interspersion pattern of the FMR1 (CGG) $)_{n}$ repeat and the haplotype distribution of closely located microsatellite markers DXS548 and FRAXAC1, in three circumarctic populations: Norwegians, Nenets and Saami. The data confirm the conservation, reported in all human populations studied so far, of an AGG interruption for each 9-10 CGG and support the stabilising effect of AGG interruptions. The data also indicate the existence of chromosomes of Asian origin in the Saami and Nenets population, thereby confirming a genetic relationship between Northern Europe and Asia. DXS548-FRAXAC1 haplotype frequencies were compared between 24 Norwegian fragile $X$ males and 119 normal males. Significant linkage disequilibrium were found between the fragile $X$ mutation and haplotype 6-4 and between normal (CGG) $n$ alleles and haplotype 7-3. European Journal of Human Genetics (2001) 9, 724-727.

Keywords: Fragile X syndrome; CGG repeats; FMR1; Saami; Nenets; Norwegian

*Correspondence: Lars Allan Larsen, Department of Medical Genetics, IMBG, Wilhelm Johannsen Center for functional genome research, University of Copenhagen, Blegdamsvej 3, DK-2200 Copenhagen, Denmark. Tel: +45 35327 497; Fax: +45 35327 845;

E-mail: lal@chrom.imbg.ku.dk or Lisbeth Tranebjærg, Department of Audiology, H:S Bispebjerg Hospital, Bispebjerg Bakke 23, DK-2400 Copenhagen NV, Denmark. Tel: +45 353163 41; Fax: +45 353139 51; E-mail: lisbetht@fagmed.uit.no

Current addresses: ${ }^{4}$ Wilhelm Johannsen Center for Functional Genome Research, Department of Medical Genetics, IMBG, University of Copenhagen, Blegdamsvej 3, DK-2200 Copenhagen, Denmark; ${ }^{5}$ Department of Medical Genetics, IMBG, University of Copenhagen, Blegdamsvej 3, DK-2200 Copenhagen, Denmark; ${ }^{6}$ Department of Audiology, H:S Bispebjerg Hospital, Bispebjerg Bakke 23, DK-2400 Copenhagen NV, Denmark

Received 8 March 2001; revised 11 June 2001; accepted 13 June 2001
Introduction

Fragile X syndrome is the most common form of inherited mental retardation, with an incidence of 1 in 4-6000 males. ${ }^{1,2}$ The condition is caused by expansion of an unstable $(\mathrm{CGG})_{\mathrm{n}}$ trinucleotide repeat sequence located in the promoter region of the FMR1 gene (OMIM no.309550). The $(\mathrm{CGG})_{\mathrm{n}}$ repeat is found as a stable inherited polymorphism in the normal population. Normal repeats ranges from 5-50 CGG, in fragile X carriers unstable premutations of 55-200 CGG are found, with an apparent overlap between stable/unstable alleles in the 40-60 repeat range. In fragile $X$ patients a full mutation of $>200$ CGG leads to silencing of the FMR 1 gene. $^{3}$ The molecular factors responsible for the transition to a premutation state are not known. However, transmission studies in fragile $\mathrm{X}$ families ${ }^{4}$ and population studies ${ }^{5}$ have indicated a stabilis- 
ing effect of AGG triplets, interspersed for each 9-10 CGG in the majority of normal $(\mathrm{CGG})_{\mathrm{n}}$ alleles.

Studies of $(\mathrm{CGG})_{\mathrm{n}}$ repeat structures in isolated human populations have added an evolutionary perspective to the understanding of the molecular factors involved in the $(\mathrm{CGG})_{\mathrm{n}}$ repeat instability. In the populations studied so far, a high degree of conservation of the AGG interruption pattern has been observed, ${ }^{6,7}$ thus supporting a stabilising effect of AGG interruptions. Here we report the internal FMR1 $(\mathrm{CGG})_{\mathrm{n}}$ sequence and the distribution of DXS548FRAXAC1 microsatellite haplotypes in three populations inhabiting the circumpolar regions of Europe.

\section{Materials and methods}

\section{DNA sample material}

Blood samples were collected from the normal population of Northern Norway and Fragile $\mathrm{X}$ patients from all over Norway. Blood samples were also collected from the Saami and Nenets ${ }^{8}$ population inhabiting the Kola Peninsula and Kara and Barents Sea coast. The persons from the Saami and Nenets populations were all questioned about the nationality of their grandparents. All tested persons had four Saami or Nenets grandparents, respectively. So far, no fragile $\mathrm{X}$ patients have been diagnosed in the Saami or Nenets population.

\section{Genetic analysis}

PCR amplification of FMR1 (CGG) $\mathrm{n}$ repeats was performed essentially as described ${ }^{9}$ using fluorescent labelled primers. DXS548 and FRAXAC1 microsatellites were amplified by multiplex PCR using FAM and TET labelled primers. ${ }^{10}$ Allele numbers were determined according to a HEX-labelled internal allelic ladder. AGG interspersion patterns were analysed by direct sequencing of the PCR fragments as described. ${ }^{10}$

\section{Results}

The number of CGG was determined in 264 Norwegian, 82 Nenets and 66 Saami normal X-chromosomes (Figure 1). The distribution of Norwegian $(\mathrm{CGG})_{\mathrm{n}}$ repeats were similar to distributions reported in Caucasian populations, ${ }^{11}$ while the Nenets population had a higher $(P<0.05)$ proportion of 29 CGG alleles compared to the Norwegian and Saami population and the Sami allele distribution displayed a broader peak around 30 CGG.

The predominant $(\mathrm{CGG})_{\mathrm{n}}$ allele in 20/59 Norwegian males (Figure 2) had an internal sequence of (CGG) ${ }_{10} \mathrm{AGG}(\mathrm{CG}$ $\mathrm{G})_{9} \mathrm{AGG}(\mathrm{CGG})_{9}$, denoted as 10A9A9 in the following. In 21 Nenets males, the most prevalent $(\mathrm{CGG})_{\mathrm{n}}$ alleles had the internal sequence of 10A9A9 (9/21) and 9A9A9 (7/21), while in 12 Saami males eight different $(C G G)_{n}$ alleles were found. Haplotypes for the microsatellites FRAXAC1 and DXS548 were constructed for 119 Norwegian, 25 Nenets and 17 Saami

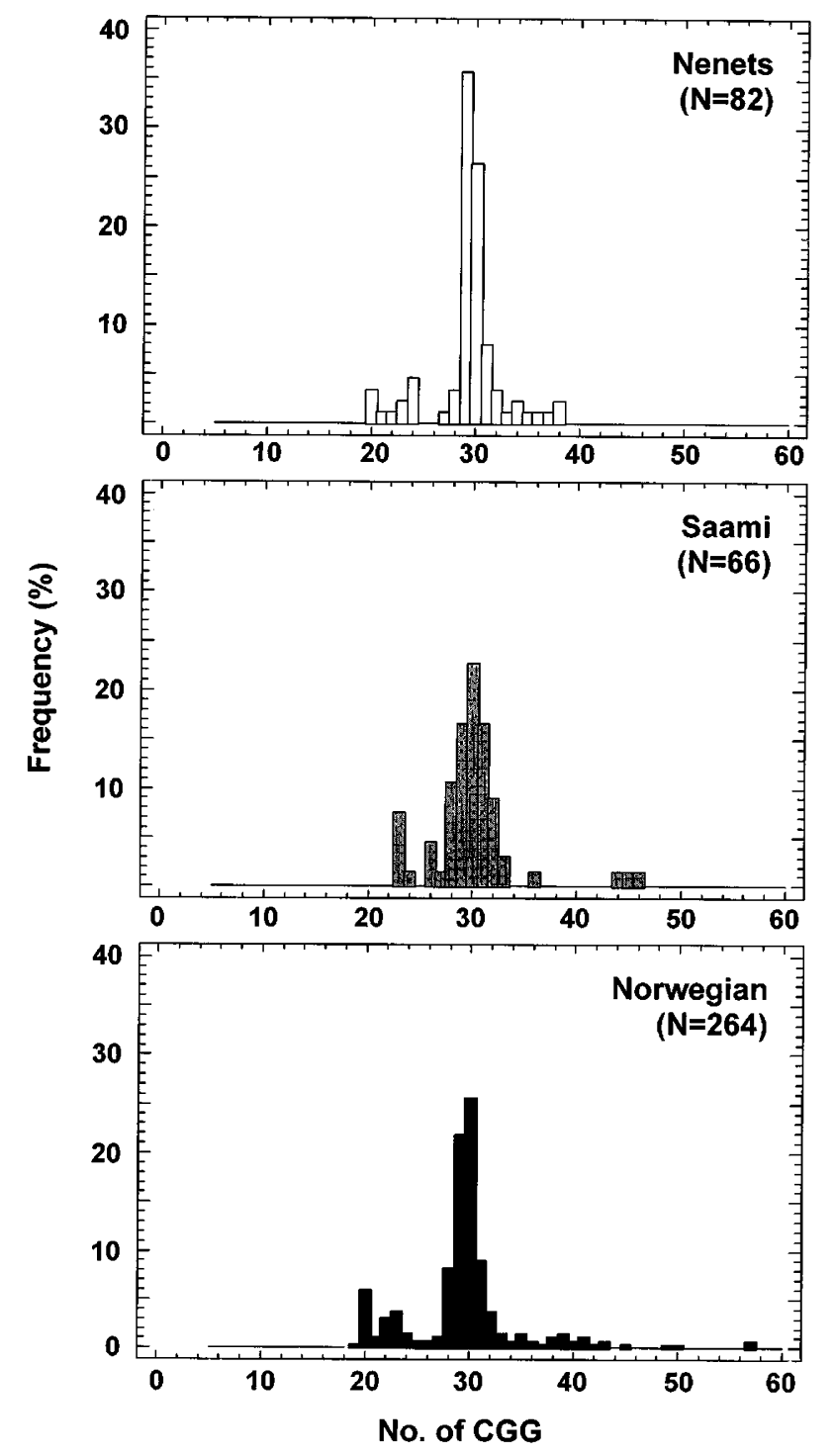

Figure 1 Distribution of FMR1 (CGG) alleles. The corresponding population and the number of alleles $(\mathrm{N})$ are shown for each distribution.

normal males as well as for 24 Norwegian fragile $\mathrm{X}$ males (Table 1). In the Norwegian normal population 14 different haplotypes were found, while only four and five different haplotypes were found in the normal Nenets and Saami population, respectively. Both in the Norwegian and the Saami population, haplotype 7-3 was predominant with a frequency of 68 and 71\%, respectively (Table 1).

However, in the Nenets population haplotypes 7-3 and 7-4 each had a frequency of $44 \%$. In the Norwegian population (Table 1) significant linkage disequilibrium was found between the fragile $X$ mutation and haplotype 6-4 $\left(\chi_{\mathrm{y}}^{2}=17.4, P<0.01\right)$ and between normal alleles and haplo- 


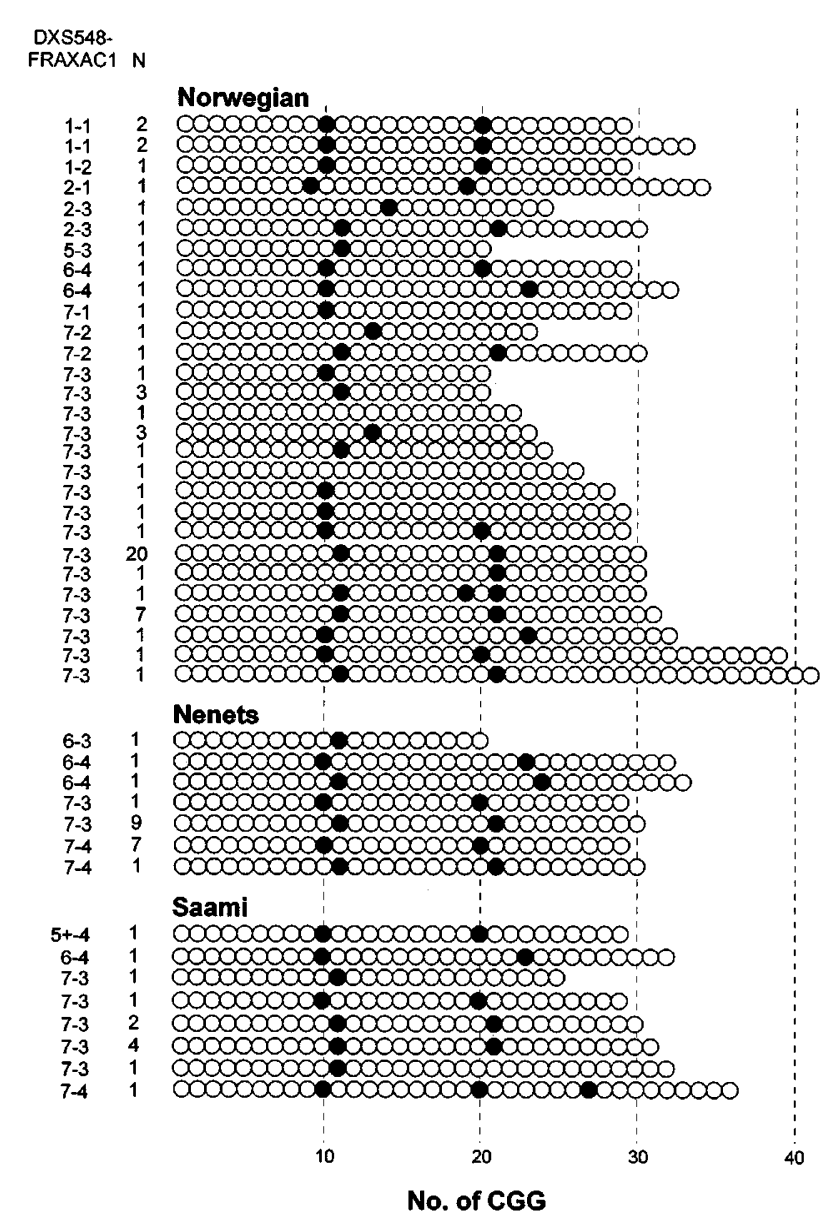

Figure 2 AGG interspersion-pattern of FMR1 (CGG)n alleles from normal males. The alleles are classified according to the associated DXS548-FRAXAC1 haplotype (first column). Only one of each allele-type is shown, and the number of each allele-type is indicated in the second column. The AGG interspersion patterns are shown from the $5^{\prime}$ to the $3^{\prime}$ end. White circles represent CGG triplets, and black circles represent AGG triplets.

type $7-3\left(\chi_{\mathrm{y}}^{2}=12.9, P<0.01\right)$, in agreement with previous studies of Caucasian populations. ${ }^{4,10,12}$

\section{Discussion}

In all three populations the most abundant alleles were 29 and 30 CGG in size and had AGG interspersion patterns of 9A9A9 and 10A9A9, respectively, as found in most populations studied worldwide. Thus, our data agree with the hypothesis that these alleles represent the ancestral (CGG) alleles in the human population. ${ }^{6}$

The most prevalent $(\mathrm{CGG})_{\mathrm{n}}$ repeat size in the Nenets population is 29 CGG (Table 1), which is more characteristic for Asian populations ${ }^{13,14}$ compared to Caucasian populations, in which 30 CGG repeats are the most common. ${ }^{10}$
Furthermore, most of the (CGG) 29 alleles found among the Nenets population were associated with haplotype 7-4, as in Asian populations, ${ }^{15}$ whereas most Caucasian (CGG) 29 alleles are associated with haplotype 7-3. ${ }^{10}$

Asian populations are also characterised by a significant shortage of $(\mathrm{CGG})_{\mathrm{n}}$ alleles of $(\mathrm{CGG})_{<26}$ compared to Caucasian populations, as well as by the presence of alleles with the internal sequence of 9A9A6A9. ${ }^{13}$ Both the Saami and Nenets population had a lower frequency of $(\mathrm{CGG})_{<26}$ alleles (9 and 13\%, respectively) compared to Caucasian populations (about 20\%), although the difference was not statistically significant. One allele with the internal sequence of 9A9A6A9 was found among the Saami population, but no such alleles were found in the Nenets or Norwegian samples. The 9A9A6A9 allele is found in approximately $20 \%$ of Greenlandic males. ${ }^{16}$

The distribution of $(\mathrm{CGG})_{\mathrm{n}}$ alleles indicates genetic differences between the Saami and the Nenets population (Figure 1). The fact that the $29 \mathrm{CGG}$ allele is the most abundant in Nenets and the presence of the 9A9A6A9 allele in the Saami indicate the presence of chromosomes of Asian origin in these populations. Our data also indicate that the degree of Asian admixture may be larger in the Nenets compared to the Saami. Although the last observation needs to be confirmed in additional studies based on a larger data set, our data are in agreement with a previous analysis of a Y-chomosomal T/C nucleotide polymorphism, suggesting a gene-flow from Asia towards Northern Europe, with a declining gradient. $^{17}$

DXS548-FRAXAC1 haplotype 6-4 was the most frequent among 24 Norwegian fragile X males (Table 1). This is similar to reports from studies of the Danish, ${ }^{10}$ Swedish and Finnish population. ${ }^{12}$ However, inter-population differences are indicated by the frequency of haplotype 2-1, which is quadrupled in Norwegian fragile $X$ males compared to the normal controls. This is similar to the Danish population, ${ }^{10}$ but different from both the Swedish and the Finnish population, ${ }^{12}$ where no differences in frequencies were reported.

In a previous study of the Danish population, $13.6 \%$ of the fragile $X$ alleles were found associated with haplotype 7-3, and it was suggested that these alleles may have originated from a pool of unstable normal alleles with the internal sequence of $10 \mathrm{~A} 19 .{ }^{10}$ No alleles associated with haplotype $7-$ 3 were found among the fragile $X$ patients in the Norwegian population, and no 10 A19 alleles were found among 59 Norwegian normal males. Thus, the results from the analysis of DXS548-FRAXAC1 haplotypes and the AGG interspersion analysis show that the fragile $X$ founder haplotypes may vary between populations and support that the CGG expansion associated with fragile $\mathrm{X}$ syndrome may originate from subpopulations of unstable alleles within the normal population. ${ }^{4}$ 
Table 1 Frequency of DXS548-FRAXAC1 haplotypes

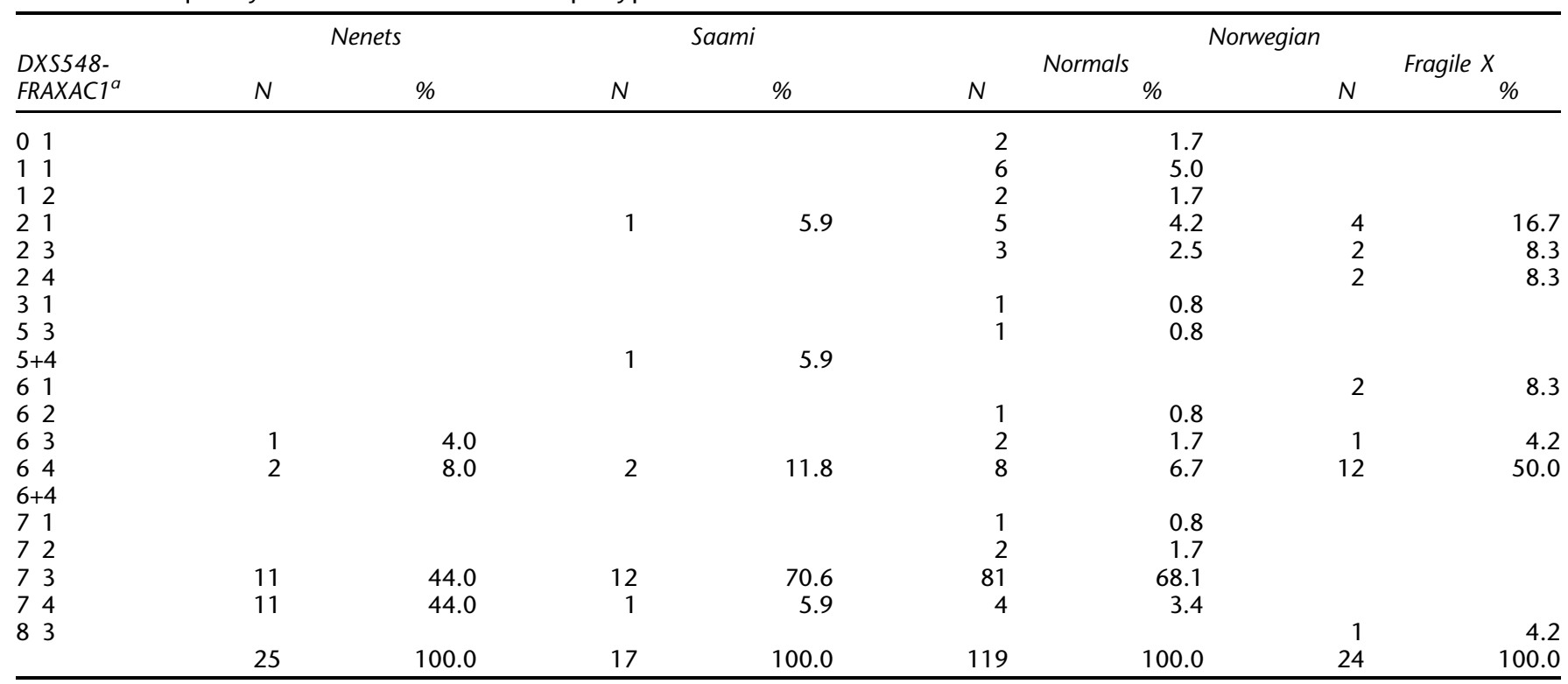

aAlleles are differing with $2 \mathrm{bp}$ in size. Alleles marked with ' + ' are $1 \mathrm{bp}$ smaller than the corresponding number (e.g. DXS548 allele $6+$ are 1 bp smaller in size than allele 6).

\section{Acknowledgements}

We would like to thank Mads Dahm Johansen for excellent technical assistance. We would also like to acknowledge the initial work done by Umayal Rubenthiran and extremely valuable ideas and discussions along the way with senior scientist, PhD Øivind Nilssen, Department of Medical Genetics, University Hospital, Tromsø, Norway. Ethical approval of the Russian part of the study was given by the Arkhangelsk State Medical Academy, Russia.

\section{References}

1 Turner G, Webb T, Wake S, Robinson H: Prevalence of fragile X syndrome. Am J Med Genet 1996; 64: 196-197.

2 de Vries BB et al: Screening and diagnosis for the fragile $\mathrm{X}$ syndrome among the mentally retarded: an epidemiological and psychological survey. Collaborative Fragile X Study Group. Am J Hum Genet 1997; 61: 660-667.

$3 \mathrm{Fu} \mathrm{YH}$ et al: Variation of the CGG repeat at the fragile X site results in genetic instability: resolution of the Sherman paradox. Cell 1991; 67: 1047-1058.

4 Eichler EE, Macpherson JN, Murray A, Jacobs PA, Chakravarti A, Nelson DL: Haplotype and interspersion analysis of the FMR1 CGG repeat identifies two different mutational pathways for the origin of the fragile X syndrome. Hum Mol Genet 1996; 5: $319-$ 330.

5 Kunst CB, Warren ST: Cryptic and polar variation of the fragile X repeat could result in predisposing normal alleles. Cell 1994; 77: $853-861$

6 Eichler EE, Nelson DL: Genetic variation and evolutionary stability of the FMR1 CGG repeat in six closed human populations. Am J Med Genet 1996; 64: 220-225.

7 Kunst CB et al: FMR1 in global populations. Am J Hum Genet 1996; 58: 513-522.
8 Prokofeva ED: The Nenetsy; in Levin MG, Potapov LP (eds): The peoples of Siberia. The University of Chicago Press, Chicago, London, 1964, pp 547-570.

9 Larsen LA, Grønskov K, Norgaard PB, Brondum NK, Hasholt L, Vuust J: High-throughput analysis of fragile X $(\mathrm{CGG})_{\mathrm{n}}$ alleles in the normal and premutation range by PCR amplification and automated capillary electrophoresis. Hum Genet 1997; 100: $564-568$.

10 Larsen LA et al: Haplotype and AGG-interspersion analysis of FMR1 (CGG)(n) alleles in the Danish population: implications for multiple mutational pathways towards fragile $\mathrm{X}$ alleles. $\mathrm{Am} J$ Med Genet 2000; 93: 99-106.

11 Sharma D, Gupta M, Thelma BK: Expansion mutation frequency and CGG/GCC repeat polymorphism in FMR1 and FMR2 genes in an Indian population. Genet Epidemiol 2001; 20: 129-144.

12 Chiurazzi P, Macpherson J, Sherman S, Neri G: Significance of linkage disequilibrium between the fragile $X$ locus and its flanking markers [editorial]. Am J Med Genet 1996; 64: 203-208.

13 Chen SH, Schoof JM, Buroker NE, Scott CR: The identification of a (CGG)6AGG insertion within the CGG repeat of the FMR1 gene in Asians. Hum Genet 1997; 99: 793 - 795.

14 Pang CP et al: Trinucleotide CGG repeat in the FMR1 gene in Chinese mentally retarded patients. Am J Med Genet 1999; 84: $179-183$

15 Poon PM et al: FRAXAC1 and DXS548 polymorphisms in the Chinese population. Am J Med Genet 1999; 84: 208-213.

16 Larsen LA et al: Analysis of FMR1 (CGG)n alleles and FRAXA microsatellite haplotypes in the population of Greenland: implications for the population of the New World from Asia. Eur J Hum Genet 1999; 7: 771-777.

17 Zerjal T et al: Genetic relationships of Asians and Northern Europeans, revealed by Y-chromosomal DNA analysis. Am J Hum Genet 1997; 60: 1174-1183. 